\title{
Videogame Agency as a Bio-costs Contract
}

\section{Pedro Pinto Neves}

HEI-LAB - Digital Human-Environment and Interactions Labs, Universidade Lusófona de Humanidades e Tecnologias, Lisboa, Portugal

nevespedropinto@zoho.com

\section{Leonel Morgado}

Department of Sciences and Technology DCeT), Universidade Aberta,

Lisboa, Portugal

leonel.morgado@uab.pt
Nelson Zagalo

Department of Communication and Art, Universidade de Aveiro,

Aveiro, Portugal

nzagalo@ua.pt

\section{ABSTRACT}

This paper presents a novel descriptive model for agency in videogames as communication. Literature pertaining to interactive works including videogames has identified the need to overcome dyadic perspectives of communication in such works. Research specifically to do with agency has called for agency to no longer be confused with freedom of action, for an integrated perspective of the player and the system, and for that relationship to be viewed as a conversation. The transactional model in this paper achieves this by proposing a nested hierarchy of levels of communication that operate as an implicit contract, negotiated between the system and the player, where the object of the transaction is bio-costs, effected through the signalling of the attainability of understandings. The paper describes research antecedents, a research agenda, the basis for the model, the model itself, examples of how the model can be used to describe videogame designs, and future research.

\section{KEYWORDS}

Videogame Design; Agency; Bio-costs; Conversation Theory; Communication.

\section{ARTICLE INFO}

Received: 02 July 2018

Accepted: 25 November 2018

Published: 13 December 2018

https://dx.doi.org/10.7559/citarj.v10i1.524

\section{1 | INTRODUCTION}

This paper proposes multiple interconnected levels of communication as a theoretical model for the analysis of the agency of videogames. The model is a means of following up on certain advances in research related to videogames and bringing them together in one framework. Chief among these advances are overcoming a dyadic perspective (alternating sender-receiver roles) of communication, holding an integrated user-system perspective, and treating agency as a conversation.

The model is an intermediate step between defining agency and deriving videogame design principles (which is mentioned as prospective future research in the Conclusion to this paper). The what already exists (as discussed in the Background section of this paper); the model in this paper deals with how agency works as communication. If the stages of that communication are not alternating signal and response (dyadic communication), then what are they, and how do they work? The levels in the model propose an answer to this question.

\section{2 | BACKGROUND}

A tendency to place responsibility for agency in playable media with either player-modelling techniques or artificial intelligence techniques has been observed and criticized in literature (Tanenbaum \& Tanenbaum, 2010; Wardrip-Fruin et al., 2009; Harrell \& Zhu, 2009). A related, recurring issue is the tendency for agency to be confused with player freedom. Janet Murray warns that "activity alone is not agency" since actions can 
have "effect, but the actions are not chosen and the effects are not related to the players' intentions" (1997, p. 128); Harrell \& Zhu warn against an "over amplification of users' freedom to act however they want" (2009, p. 46); and Tanenbaum \& Tanenbaum explain that "unrestricted player actions" are not "sufficient for meaningful play" (2010, p.14).

Agency has been redefined by Wardrip-Fruin et al. (2009) and by Tanenbaum \& Tanenbaum (2010) in ways that avoid misplacing responsibility for agency and misconstruing agency as player freedom. The redefinition in Wardrip-Fruin et al. (2009) is viewed by Tanenbaum \& Tanenbaum as having arrived "at complimentary conclusions" to their own (2010, p.12). Both of these redefinitions carry an integrated perspective of the player and the system, where neither the player nor the system can be fully isolated from one another; agency does not fall on neither the system nor the player, but encompasses them both as one, and extends beyond them - agency is real, and, to some extent, independent of both system and player. WardripFruin et al. distinguish their perspective "from previous presentations of agency as an audience experience or structural property of works" (2009, p. 8). Tanenbaum \& Tanenbaum see "designers and performers" as being "in a type of conversation with each other, mediated by the game" (2010, p. 14). As for agency being misconstrued as player freedom, Wardrip-Fruin et al. emphasize enticing "players to desires the game can satisfy" (2009, p. 7), and Tanenbaum \& Tanenbaum emphasize meaning, which shifts "the emphasis in an interaction away from the outcome of a choice and towards the intent which underlies that choice", thus avoiding the construal of "agency as freedom" (2010, p. 14).

An issue related to the need for an integrated perspective is how communication perspectives (e.g. semiotics) tend to be dyadic, alternating between signal and response - a "transmission model" that "breaks down" when applied to videogames, as noted by Upton (2018, p. 8). Aarseth makes a comparable criticism of "traditional" (1997, p. 22), "chain of signifiers" (1997, p. 24) models with regard to their application to cybertext.

Outside of studies of the agency of playable media, the need for an integrated perspective is also present. Research on Emergent Narratives as a resolution to the Narrative Paradox arrived at the need for Purposeful Authoring (Louchart et al., 2008), which takes into account both player and system. The notion of a contract of interactive storytelling (Young, 2002; Adams, 2006) was a way of circumventing the "Problem of Narrative Flow" (Adams, 2013, p. 96) of how to split responsibility for plot coherence and the quality of storytelling between player and the design of the system.

An example of a perspective of videogames that is anchored on the system - as opposed to being integrated in the meaning of this paper - is the MDA (Mechanics, Dynamics, Aesthetics) framework (Hunicke et al., 2004). MDA allows games to be approached from both the direction of mechanics "at the level of data representation and algorithms", or aesthetics at the level of evoking "emotional responses" in the player (Hunicke et al., 2004, p. 18). However, approaches from either direction must always go through the intermediate level of dynamics, which Hunicke et al. (2004) explicitly equate with the system.

An example of a perspective of videogames that is anchored on the player is Situational Design (Upton, 2018). Situational Design touches on the game as a system in its concepts of Situations, Constraints, and Moves, but is explicitly stated to be "a player-centric approach" (Upton, 2018, p. 6) to overcome an observed tendency to abstract the player in most game design approaches. Upton is concerned with giving coverage to aspects of play, similar to the model proposed in this paper, but emphasizes concepts of playfulness and anticipation in meaning-making, whereas this paper is more concerned with agency as communication, and its requirement of an integrated - as opposed to player-centric - perspective.

\section{3 | BASIS FOR THE MODEL}

To deal with the issue with dyadic models of communication, the model in this paper draws from behavioural-role pragmatics (Watzlawick \& Beavin, 1967), in that overcoming the communication dyad requires an appropriate unit of analysis. The functioning of the model in this paper is drawn from Conversation Theory (Pask, 1976). This is a means for the model to delve into how the conversation of agency in Tanenbaum \& Tanenbaum (2010) is carried out. Following Conversation Theory, the 
model in this paper sees agency as a contract between the player and the system. Conversation Theory is an approach from second-order cybernetics, that is, from a cybernetics of cybernetics, or a cybernetics of observing systems as opposed to observed systems (Pangaro, 2002) - a cybernetics that deals with subjectivity where first-order cybernetics is concerned with control. In keeping with this second-order cybernetics approach, the unit of analysis used to build the model in this paper draws from the concept of biocost (Dubberly et al., 2009) - which is also from second-order cybernetics - as to ensure compatibility between the unit of analysis of the model and the model's functioning as a contract as drawn from Conversation Theory.

The model proposed in this paper is transactional. Upton criticizes widespread "transactional" approaches to videogame design $(2018$, p. 8$)$ as excessively focused on activity. Upton's Situational Design moves away from this. As mentioned in the Background section of this paper, we argue Upton's player-centric approach to be incompatible with our focus on agency as a conversation. Instead of moving away from a transactional approach, we change the object of the transaction to bio-cost to be consistent with the Contract-view from Conversation Theory. This lets our contract-model operate in terms of negotiating the anxiety from having too many or too few possibilities in interpretation of the system, similar to what is described in Carvalhais \& Cardoso (2017).

Behavioural-role pragmatics is a triadic model of communication, which overcomes the artificial "punctuation" of the communicative exchange as sender-sign or sender-receiver (dyadic) relation between participants in the exchange (Watzlawick \& Beavin, 1967, p. 7). Watzlawick \& Beavin instead see "any behavioral event in a sequence" as "a stimulus for the event which follows it, and both response and reinforcement to the one which preceded it", with this serving "as the minimum complexity of any interchange" (Watzlawick \& Beavin, 1967, p. 7). These behavioural roles are the unit of analysis that allows behavioural-role pragmatics to overcome a dyadic model of communication. Through its own unit of analysis, the model in this paper provides this for agency as communication in videogames - a behavioural-role that shifts according to the different levels of observation in the model.

Conversation Theory sees the participants in a conversation "as part of a contract" agreeing "to obey the rules of the conversational language L", where "the participating agent makes sure that the L syntax is respected" (Pask, 1976, p. 4). Understanding is a structural property of conversations, where a topic is understood and is concretely made understandable (becomes an attainable "understanding") if that topic "is explained" and "can also be derived from other topics in the conversational domain", inasmuch as these derivations are explanations of explanations, or a "systematic justification of an explanation" (Pask, 1976, p. 4). The contract-model in this paper sees agency as exchanges in the attainability of understandings, through bio-cost.

The concept of bio-cost is meant to help "map the cognitive transitions that are the core value of using computers or any human activity" (Pangaro, 2008, p. 36). Bio-cost then, consists of "effortminimization trade-offs" (Pangaro, 2008, p. 37) in the context of such activities - a "second-order awareness of the toll that a task is taking", which "becomes part of a feedback loop" that helps endusers "estimate the bio-cost expenditure required to be successful" (Dubberly et al., 2009, p. 188). These trade-offs are involved in how "we humans create and evolve our goals in real-time and in parallel to our actions and to other goal-related 'mental processing"', where "we negotiate with ourselves and others in attempts to lessen or increase some attributes of desired outcomes above others" (Pangaro, 2008, p. 37). Bio-costs are the currency of agency in videogames in the model in this paper. As per the model, players play by expending bio-costs following their hypotheses for what the system is like. When these hypotheses prove correct, the player gets a return of investment in bio-costs, which can then be re-invested towards further returns. These returns and investments are negotiated by the user and the system through the contract of agency in the model, through the different levels of abstraction in the model.

\section{4 | THE MODEL}

As can be seen in Figure 1, the model proposed in this paper consists of a nested hierarchy of eight levels of abstraction. Agency - as commitments to 


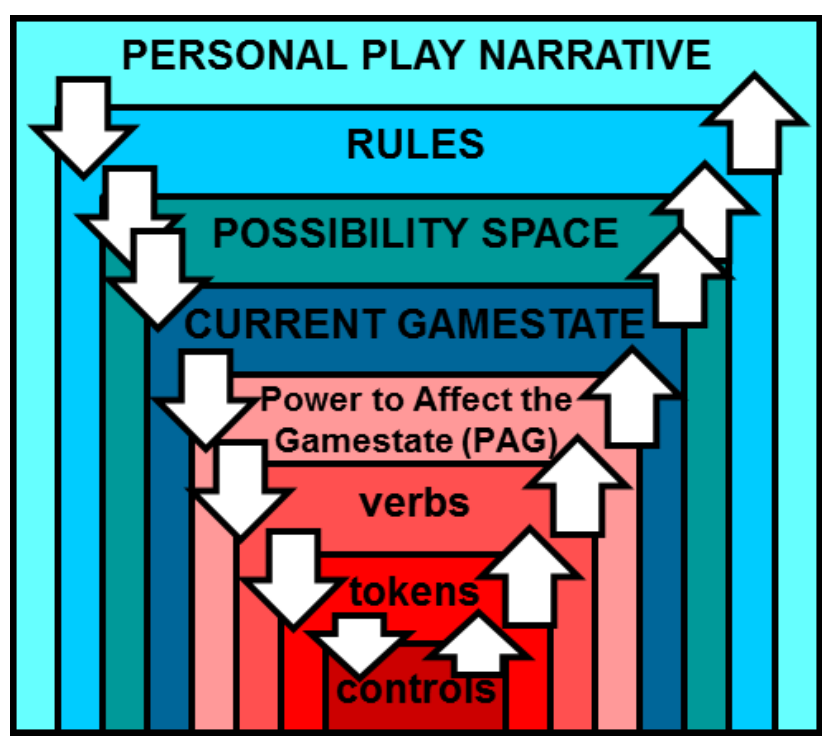

Figure 1 | The eight levels of the model in this paper.

meaning (as per Tanenbaum \& Tanenbaum, 2010) - is collaboratively produced between levels in videogame play by the player and the system, starting at the bottom of the structure (the Controls level), and working its way up to the top of the structure (the Personal Play Narrative level).

In Tanenbaum \& Tanenbaum (2010), actions taken by the player in the course of interacting with the videogame can be seen as speech acts, and matter because of their illocutionary point - i.e., what they mean. For its part, the system also undertakes speech acts in how it expresses a gameworld, in how it provides constraints to the player's actions, and what are the expectable results of those actions. The speech acts of the player and those of the system are not isolated from each other; they are instead derived from each other (as part of a conversation). Figure 1 shows eight levels of abstraction for this mutual commitment to meaning, where understandings are derived following Conversation Theory (Pask, 1976) between levels, and are used to negotiate bio-costs between the user and the system.

Speech acts in the model define terms for exchanging promissory notes towards bio-cost replenishment between the player and the system of the videogame. The speech acts of the system offer play possibilities. Through her speech acts, the player loses interactional options by committing to certain possibilities and not others. Together, these form a process of commitment to meaning that produces agency. The time a player spends playing a videogame and keeps coming back to that videogame is a continual contract renegotiation, done for its own sake. Bio-costs are both what is being negotiated and the fuel that powers the player and the system taking part in the negotiations.

The player implicitly knows that taking on bio-costs along certain lines will lead to a corresponding replenishment - or repayment - of those bio-costs along those same lines. Moreover, that repayment will carry a profit margin of heightened skills or improved parameters for interaction, lowering the toll of bio-costs in subsequent tasks, and letting the player take on higher and higher bio-costs as she moves along in playing the videogame.

For its part, the system benefits from bio-costs due to uncertainty being taken away as to what the player might try to do next in the context of the videogame. This narrows down the possibilities for action the system has to offer, and relieves the system of having to be infinitely complex. Every time the player takes on a new bio-cost, the system is relieved of having to cater to some aspect of meaningless freedom of action.

The benefits for the player and the system in negotiating bio-costs in a contract of agency match the views and goals of the definition of agency in Wardrip-Fruin et al. (2009) and in Tanenbaum \& Tanenbaum (2010) - namely as it relates to overcoming the conflation of agency with freedom of action, and achieving an equal distribution of responsibility for agency between the player and the system.

\subsection{THE LEVELS IN THE MODEL}

In the model, following Conversation Theory (Pask, 1976), understandings are signaled in each level, and become meanings in the next level. On that next level, the meanings inform new understandings, which lead to a commitment on the level above that, and so forth. Bio-cost is what is used for alternately signaling understandings and committing to meanings. In the model, successful functioning of the videogame is understood as meaning happening at each and every one of these levels; videogame design problems are understood as certain levels being skipped or their meaning being obfuscated by other levels, disrupting the flow of commitments. Solutions to videogame design problems offset such effects by tweaking the 
construction of meaning in certain levels. Situations of this happening in games can be found in the Examples section of this paper. Play in videogames is defined as the time between the system creating a need for interactional support and the player running out of need for this support. This is the most abstract, highest level in Figure 1 - the Personal Play Narrative.

As per the model in Figure 1, the player and the system implicitly agree on three conditions. The first condition is that understandings exist in the context of their conversation - the playing of the videogame. This means that the videogame is finite and non-arbitrary. The second condition is that the understandings are attainable. This means that that the depth and breadth of the player's range of actions has assuredly been designed to be tractable to some degree of comfort and reliability, given the conditions of play. The third condition is conversational parsimony from both the system and the player. This means that no understandings go to waste - any understanding is valuable in signalling further understandings. The system can rely on the player not skipping ahead in the course of their conversation without first attaining the requisite understandings. The player can rely on the system not turning up understandings that are not supported by preceding understandings.

The Controls level (bottom of Figure 1) is the terms for the bio-cost transactions in the videogame. More than simply input or commands, controls in this model can be understood as the letter of the contract - they are the most elementary form of signalling that there are attainable understandings. The controls phrase bio-costs - they are the unit of currency to bio-costs traded at levels above Controls in the model.

The next level in Figure 1 refers to Tokens objects in the gameworld that register and store quantities of bio-costs by changing state; they make those quantities available and visible by being placed across the gameworld in a certain distribution. This profile of distribution is relevant to defining a play aesthetic. A token in this model is not just any object with placement in the gameworld, even if this object is interactive; it needs to make a difference to the sale (expenses, returns) of bio-costs in the gamestate - otherwise the object counts as a prop.
Next in Figure 1 are Verbs, which are the bio-cost transactions themselves - a control (terms for the transaction) activated on a token (quantity in the transaction). The player positions herself in the conversation - i.e., the conversation in which the contract is negotiated - through verbs. Verbs let her expend bio-costs (take on uncertainty and stress, employ attention, cognition, motivation), effect bio-cost savings, and re-invest profits. A verb with tweaked, strengthened power to affect the gameworld (e.g., powered-up, levelled-up), or employed with acquired skills by the player is a biocost profit being re-invested. A verb that changes to automate a previously manually-entered sequence of inputs is a bio-cost saving.

Above Verbs is Power-to-Affect-the-Gamestate (PAG), as the accrued sale of bio-cost transactions - what the player ventured and gained in playing the game. Controls, Tokens, and Verbs result in PAG. PAG is the point of wielding Controls, Tokens, and Verbs, and the means whereby the system empowers the player to play the game, and provides a conversational point to playing the game.

Following PAG is the level of the Current Gamestate, for what results from all the transactions and signaling below. Bio-cost transactions keep taking place, and they actualize (make Current) a particular gamestate, then another, then another, and so forth. The Current Gamestate is a given point in the process of contract negotiation between the system and the player, which helps them decide how to move forward in the negotiation.

The Possibility Space holds all the alternate potential versions for future Current Gamestates where the conversation can go from a given Current Gamestate. This space is perceived as the player's sketched-out mental model of all the potential bio-cost sinks and payoffs in all the untapped playable content in the game by that point in the negotiation. The player does not need to experience every possibility; only find one legitimate conversational route across the negotiation - a chain of commitments.

The Rules level is what makes the Possibility Space finite, and therefore lets it fit in the conversation. The system is spared from having to specify everything that has been learned in the 
conversation with each new topic. Without Rules, the player would not be able to distinguish further possibilities from her current gamestate, due those possibilities being infinite.

The Personal Play Narrative is the player's mental model of the entire negotiation process. It's there from the moment the player first picks up the videogame, right until she drops it. These are the stories we tell ourselves about playing games e.g., I was getting stuck, but then I saw the way forward; I was finding the game too hard, but then it occurred to me that.... In the model, these stories are about bio-costs and committing to meaning.

\subsection{EXAMPLES}

In this section, the model in this paper is used to describe game design in two different published commercial off-the-shelf videogame titles. For each title, the model is first used to describe aspects of the videogame's design as potential threats to the maintenance of agency as a conversation. This means risks of the player losing herself from the conversation, or of holding an inaccurate mental model of the system which would only become more inaccurate with time. Next, the model is used to describe other aspects of each videogame which counteract the threat to agency.

The first title to be examined is Fallout 2 (Interplay Productions, Inc., 1998). Fallout 2 (FO2) is a roleplaying game where the player defines a character by balancing a series of parameters previous to the gameworld becoming accessible. The gameworld offers problems which measure this balance of parameters against virtual dice rolls. Successfully solving problems affords the player the means to strengthen her character against more demanding, future problems. There are roughly three styles of problem-resolution in FO2, which can be described as diplomacy, subterfuge, and combat. Consistently succeeding with any one style requires sustained commitment strengthening the balance of parameters towards one style while weakening the others in the face of limited opportunities to improve characterparameters. When an attempt to solve a problem using the diplomacy style or the subterfuge style fails, the player is often forced into combat. A character geared towards diplomacy or subterfuge then incurs an opportunity-cost from having weakened its combat-style. This can be regarded

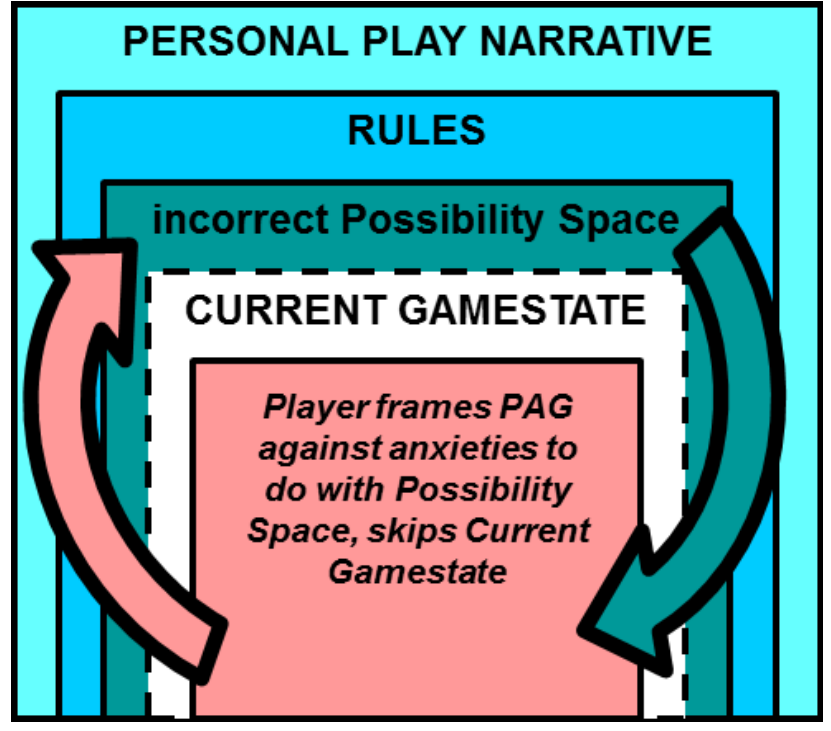

Figure 2 | Threat to agency in FO2.

as a legitimate design choice for reasons such as e.g. differentiating stakes between play-styles towards roleplaying.

From the standpoint of the model in this paper, defaulting to combat in FO2 can incorrectly signal the nature of the system at the level of the Current Gamestate in the model. The player might feel that the system is signalling that her bio-cost investment in the subterfuge or diplomacy styles - the effort in continually interpreting the possibilities in the system and the anxiety of trying to make conversationally-relevant choices - is less valid. This is not necessarily the case. The character might be within the parameters to succeed at that particular subterfuge or diplomacy attempt, and the failure might be due to virtual dice-rolls on the part of the system, and could have just as well been a success. Even if the character was outside the parameters, sustaining the investment in the diplomacy or subterfuge styles might still yield a competitive character.

In the model, the player should always evaluate her Power to Affect the Gamestate (PAG) against the Current Gamestate, and from that Current Gamestate derive the Possibility Space for subsequent play against the Rules and so forth. The defaulting to resolution by combat in FO2 can cause the player to skip the Current Gamestate and evaluate her PAG against the Possibility Space, as shown in Figure 2. In other words, she is not evaluating her diplomacy or subterfuge PAG against the actual demands of the system; she is looking at an incorrect Possibility Space (due to 


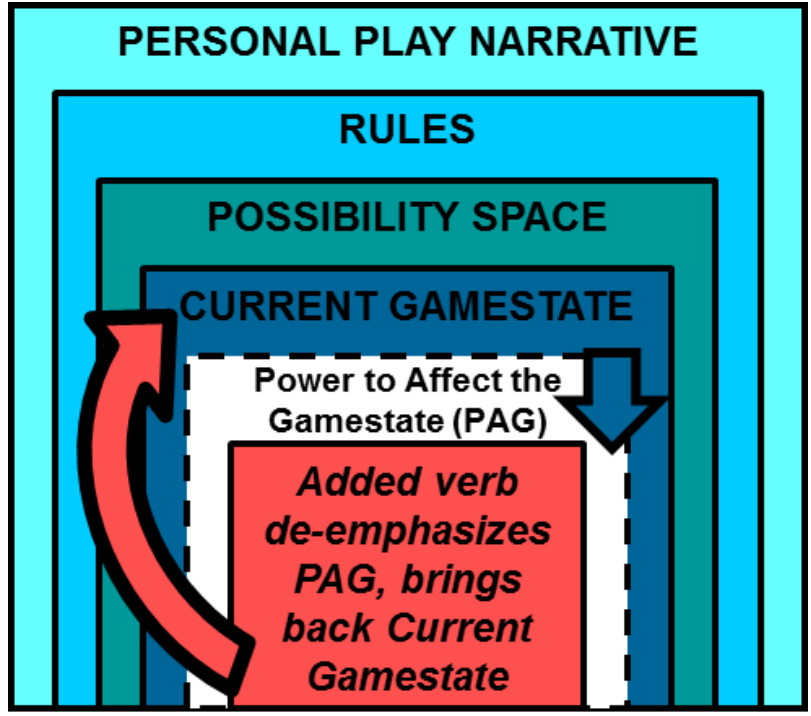

Figure 3 | Due to 'insurance' Current Gamestate is not skipped.

skipping the Current Gamestate level) where all her future attempts at resolution default to combat. She will either discard her character and start over, or start unnecessarily investing in a combat style.

FO2 features a Targeted Shots verb. This verb raises the stakes of a combat action - the player takes on increased chances of failure against more expressive effects in case of success. Using the verb for making a targeted shot is an alternative to the more standard non-targeted verb, which yields more consistent and less expressive results. Character-parameters that help success in targeted shots can overlap with parameters that help the diplomacy and subterfuge styles of resolution. Making the most out of the more standard, nontargeted verb requires a different investment, more geared towards the combat style - e.g. being able to survive enemy attacks long enough to enjoy the consistent results of the non-targeted verb.

From the standpoint of the model in this paper, the targeted shot verb remedies the threat to the contract of agency from defaulting to combat. This verb works as an insurance policy for the bio-costs the player has already invested and will invest in the future in the diplomacy style or in the subterfuge style. The system is signaling to the player that although her investment is riskier, she can expend more bio-costs (anxiety, having to evaluate things more carefully) in the targeted shot verb to get insurance against defaulting to combat. Her investment in the other styles - due to the overlap in parameters between targeted shots and the non-combat styles - can still pay off. The system gets an added means for explaining biocost returns to the player. As seen in Figure 3, improved signaling at the level of verbs helps deemphasize PAG, which prevents it from obscuring the Current Gamestate, which would have made the player skip that level.

The other title to be examined is Red Faction: Guerrilla (THQ Inc., 2009). In Red Faction: Guerrilla (RFG), the player learns to roam a succession of sandbox maps and quickly pick out and engage activities. The activities tend to be relatively shallow - the player moves in, quickly forms a plan no more complicated than causing as much destruction as possible as quickly as possible, implements it, and moves on. This is a steady process of accumulating resources and transforming the gamestate towards desired outcomes. Part of how the system trains the player in this shallow and wide attentional footprint is good interactional support. A map can be quickly pulled up which shows distribution of resources in the gameworld. The player can select a point in the map, which will create a route towards that point. This route appears both in 2D on the map and projected in 3D on the gameworld. The route dynamically alters to always show the optimal path to the destination, even if the player goes off-course. The player quickly grows accustomed to setting routes, changing her mind halfway, easily dismissing routes and setting new ones.

Occasionally the system will insert variants into sandbox play in RFG. These are higher-stakes, moving targets, available only for a limited time. Instead of resources being evenly distributed across the gameworld to be accessed at the player's leisure, the player has to drop what she is doing and chase down a spike in resource availability, or incur an opportunity cost. The variant play instances themselves also require more planning and have riskier execution compared to established play. The attentional footprint suddenly shifts from wide and shallow to narrow and deep. Both the standard wide and shallow footprint and its variant are legitimate design choices. The standard footprint emphasizes the spatial and simulational qualities of the system, while the variant prevents play from becoming a chore. 


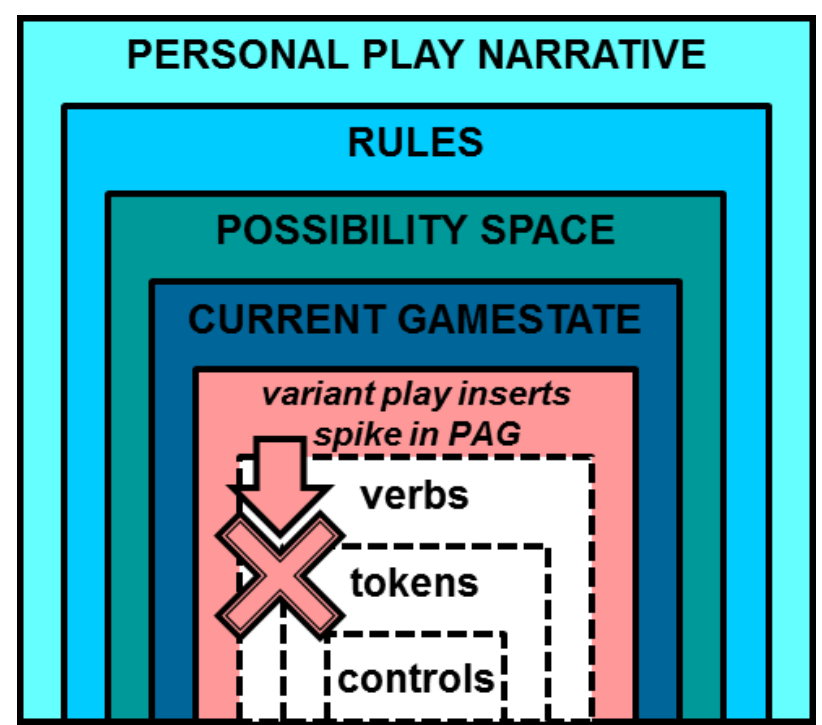

Figure 4 | PAG becomes disconnected from tokens and controls.

From the standpoint of the model in this paper, the shift from a wide and shallow attentional footprint to a narrow and deep attentional footprint in RFG can cause a disconnect between the level of Power to Affect the Gamestate (PAG) and the levels below of Verbs, Tokens, and Controls. The player is accustomed from the wide and shallow footprint to relatively lighter investments of bio-costs in Verbs, Tokens, and Controls against smaller but steadier returns in PAG. The targets on the map are stationary and always available, which makes play more forgiving. The shift to a deep and narrow footprint increases the PAG to be gained in one instance of play, but also the loss of PAG from mistakes. The player feels cheated in the contract, and stops trusting her ability to invest bio-costs at a low-level. This disconnection is illustrated in Figure 4.

Whenever an instance of variant play emerges in RFG's sandbox, the system does something that is seemingly counter-intuitive - it sabotages its own interactional support. In standard play, the player grows accustomed to taking the lead in handling the means of interactional support, laying down routes and selecting objectives at her leisure, without any consequences for dawdling or changing her mind. In variant play however, the system subverts the player's control of the map, and forces her to engage the activity in the system's terms. These terms are dynamically set by the system, as a moving target proceeds along a route, circumventing obstacles, and forcing the player to catch up. The laying of routes, which is normally exclusive to the player in sandbox play, is

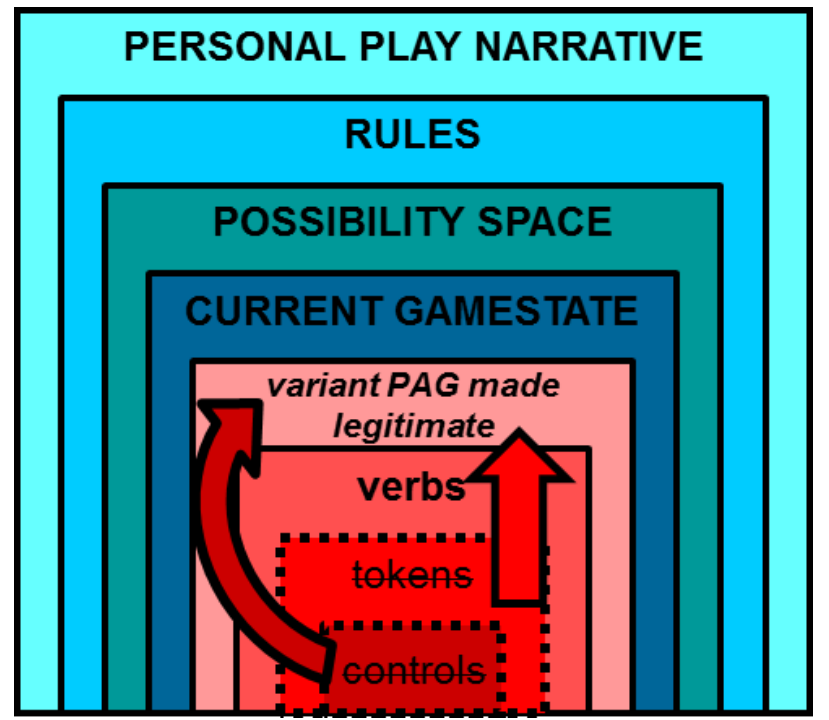

Figure 5 | Update to PAG in variant play is anticipated.

hijacked by the system. A different-coloured route is laid down on the map without the player's input. The system's red route is even signified to have higher priority relative to the player's yellow route the player is asked whether she wants to press a button to slave her yellow route to the objective moving along the red route.

The moving target in variant play is a concentrated pool of resources. It represents an opportunity to either gain an unusual amount of PAG, or pass it up. If the player misses it, she will feel as if she has just lagged behind in the process of jointly constructing meaning with the design in conversation. This entails growing her capital of bio-costs for conversing with the game, and in RFG's case involves developing the playercharacter and evolving the gameworld by acquiring different kinds of resources, and becoming more skilled at the game.

From the standpoint of the model, RFG sabotaging its own scheme of interactional support remedies the threat to agency from shifting between attentional footprints. It anticipates the shift, and gives the system an additional means of signaling the shift. Tokens (map icons and objects in the gameworld) no longer wait for the player. Controls that were exclusive to the player (route-laying) are made available to the system and wielded by the system. Without this degradation in tokens and controls, the player might only feel the shift in attentional footprint when she got close to the moving target from variant play. With the degradation, she can feel the shift no matter how 
far she might be from the target when variant play becomes available.

As illustrated in Figure 5, the terms for bio-cost (controls) and the storage of bio-cost (tokens) shift to signal a change in the sale of bio-costs (PAG) under negotiation in the contract. If only the sale changed, or changed without warning, the player would feel cheated; instead, the player is apprised of changes to the values that will add up to that sale.

\section{5 | CONCLUSION}

The model in this paper contains eight different levels of abstraction for how agency works as communication in videogames. The model is meant to provide a follow-through on certain currents in agency research. The levels - in what they are and how they work together - describe how the player uncovers the system as intent and how the system makes itself discoverable, from low-level to highlevel. The processes in the model - signaling of the attainability of understandings, investments of biocost against assurances of returns - let the model meet the objectives (stated in the Introduction of this paper) of not seeing levels of communication as a sender and receiver dyad, as well as holding an integrated perspective of the system and the player in agency, and seeing agency as a conversation.

The model is intended as an intermediate step between theory for the phenomenon of play and actionable design principles derived from that understanding. As mentioned in the Background section of this paper, the what is relatively wellestablished, across different perspectives and approaches, including agency; this paper means to make a contribution to the how.

The process of meaning-making that concerns the model in this paper is similar to what Carvalhais \& Cardoso (2017) describe as the Virtuosic Interpretation that users of processor-based artifacts engage in. Of particular interest is the anxiety experienced by these users by having too few or too many possibilities, as determined by how much accuracy can be had in user mental models of the system. This matches the functioning of the model in this paper (with regards to bio-cost), as well as the definition of agency in Wardrip-Fruin et al. as "a phenomenon, involving both the game and the player, that occurs when the actions players desire are among those they can take as supported by an underlying computational model" (2009, p. 7). Carvalhais \& Cardoso (2017) recommend exposing the computational-model in the artifacts themselves to account for Virtuosic Interpretation, and affirm the value of the iterative process in designing processor-based artifacts. An intermediate step such as the how in the multiple levels of the model in this paper - is warranted. It will enable a broader set of design strategies for uncovering the underlying computational model, beyond outright exposing it in artifacts. The model can also guide iterative design.

Future research following from the model in this paper includes empirical validation of the model's predictive capabilities. The examples for how the model can be used to describe videogame design in this paper take two finished, published titles and frame their design in the model. These examples inform the argument for the model's validity, which is made in the rest of the paper. In the examples, aspects of the design that can compromise agency from the standpoint of the model are described, as well as aspects which prevent that harm to agency. Future research will instead look to unfinished designs in ongoing videogame development projects. The model already provides an integrated videogame-specific second-order perspective of the phenomenon of play; that is, what happens in play outside of explicit activity as well as inside explicit activity. In an unfinished, ongoing iterative design process, the model would yield design rules and even patterns. This will be done iteratively. The model will be applied in detecting potential design problems before they emerge in playtesting. Should the predictions be confirmed in playtesting, the model will have to be used to determine solutions for those design problems, to be evaluated in subsequent iterations and playtesting. Otherwise, the application of the model will have to be refactored, until predictions of the design problem and the corresponding solution are achieved.

Further subsequent research in the model lies with design tools and vocabularies - using the model to encapsulate a wide variety of problem-solution pairs in videogame design, or to derive actionable design principles, or redefine existing principles. Empirical testing of predictive capabilities requires projects that are already ongoing - pinpointing a 
design problem in particular levels in the model, and doing the same for a solution. The next step would be to create novel videogames entirely from the model, to test and illustrate design principles. This would be a move from loose design rules from ongoing projects, to comprehensive pattern collections, tools, and vocabularies, from fresh projects.

\section{ACKNOWLEDGEMENTS}

The research for this work was supported by the Portuguese FCT (Fundação para a Ciência e Tecnologia) through grant SFRH/BD/76365/2011.

\section{REFERENCES}

Aarseth, E. (1997). Cybertext: Perspectives on Ergodic Literature. Baltimore: The Johns Hopkins University Press.

Adams, E. (2006). A New Vision for Interactive Stories. Lecture delivered at the Game Developers' Conference, San Francisco, California, USA.

Adams, E. (2013). Resolutions to some problems in interactive storytelling. Teeside University, Middlesborough, England, UK.

Carvalhais, M., \& Cardoso, P. (2017). Creation of meaning in processor-based artefacts. ISEA 2017: International Symposium on Electronic Arts, Manizales, Colombia.

Dubberly, H., Maupin, C., \& Pangaro, P. (2009). Bio-cost An Economics of Human Behavior. Cybernetics and Human Knowing, 16(3-4), 187194.

Fallout 2 [Computer Game]. (1998). Irvine: Interplay Productions, Inc.

Harrell, D. F. F., \& Zhu, J. (2009). Agency play: Dimensions of agency for interactive narrative design. In Proceedings of the AAAI 2009 Spring Symposium on Narrative Intelligence II. Menlo Park: AAAI Press (pp. 44-52). Retrieved from http://www.aaai.org/Papers/Symposia/Spring/2009/ SS-09-06/SS09-06-008.pdf.

Hunicke, R., LeBlanc, M., \& Zubek, R. (2004). MDA: A Formal Approach to Game Design and Game Research. AAAl-04 Workshop on Challenges in Game Al (Vol. 4, No. 1, pp. 17-22).
Louchart, S., Swartjes, I., Kriegel, M., \& Aylett, R. (2008). Purposeful authoring for emergent narrative. In Lecture Notes in Computer Science (including subseries Lecture Notes in Artificial Intelligence and Lecture Notes in Bioinformatics) (Vol. 5334 LNCS, pp. 273-284). http://doi.org/10.1007/978-3-540-89454-4_35.

Murray, J. H. (1997). Hamlet on the Holodeck. Cambridge, MA, USA: MIT Press., pp. 273-284). http://doi.org/10.1007/978-3-540-89454-4_35

Pangaro, P. (2002). New order from old: The rise of second-order cybernetics and implications for machine intelligence. American Society for Cybernetics in Vancouver.

Pangaro, P. (2008). Instructions for design and designs for conversation. In Handbook of conversation design for instructional applications (pp. 35-48). IGI Global.

Pask, G. (1976). Conversation Theory Applications in Education and Epistemology. Amsterdam: Elsevier.

Red Faction: Guerrilla [Computer Game]. (2009). Agoura Hills: THQ Inc.

Tanenbaum, K., \& Tanenbaum, J. (2010). Agency as commitment to meaning: communicative competence in games. Digital Creativity, 21(1), 1117. http://doi.org/10.1080/14626261003654509.

Upton, B. 2018. Situational game design. CRC Press.

Wardrip-Fruin, N., Mateas, M., Dow, S., \& Sali, S. (2009). Agency Reconsidered. Breaking New Ground: Innovation in Games, Play, Practice and Theory. Retrieved from http://www.digra.org/wpcontent/uploads/digital-library/09287.41281.pdf.

Watzlawick, P., \& Beavin, J. H. (1967). Some formal aspects of communication. The American Behavioral Scientist, 10(8), 4-8.

Young, R. M. (2002). The Cooperative Contract in Interactive Entertainment. In Socially Intelligent Agents (pp. 229-234).

\section{BIOGRAPHICAL INFORMATION}

Pedro Pinto Neves holds a Master in Arts in Digital Game Design from the University College for the Creative Arts at Farnham, UK and a PhD in 
Communication Sciences, for which he submitted a thesis on Videogame Design and Agency (Universidade do Minho, 2017). He is an Auxiliary Professor at ULHT (Universidade Lusófona de Humanidades e Tecnologias, Lisboa) where he teaches courses on Interative Narratives and on Game Design. His research interests are videogame design vocabularies and conceptual tools. He is a member of HEl-Lab.

Leonel Morgado is Assistant Professor with Habilitation, at Universidade Aberta (Portuguese Open University), a public university focusing on elearning, where he lectures on programming and the use of virtual worlds. His main research interest is the use and development of virtual worlds as tools for learning and business, which he pursues since 2000 , focusing on multi-user platforms since 2006. He authored over 100 papers, in journals, conferences, and as book chapters. Before pursuing an academic career, he was business and technical manager of an hardware import, distribution, and retail company, terminologist for the localization teams of MS Office 97 and Oracle InterOffice, language consultant for IBM/Lotus, a coordinator of Web-development and softwaredeployment teams, and manager of a cooperative extension team fighting the digital divide in rural villages.

Nelson Zagalo is Associate Professor at University of Aveiro - Portugal. Got his PhD in Communication Technology on interaction design and entertainment experience. Created a master program on Interactive Media; founded the laboratory EngageLab; founded the Portuguese Society for Videogames Sciences. $\mathrm{He}$ is an integrated researcher at Digimedia - Digital Media and Interaction. Nelson has published "Interactive Emotions, from Film to Videogames" (2009) and "Creativity in the Digital Age" (2015). 